\title{
Effect of the Monthly Injectable Combined Contraceptives versus Oral Contraceptive Pills on Mood
}

\author{
Ghada M. Khafagy ${ }^{1, *}$, Hebatallah L. Shalaby ${ }^{2}$, Nagwa E. Saad ${ }^{3}$, Marwa D. Hasan ${ }^{1}$ \\ 'Department of Family Medicine, Faculty of Medicine, Cairo University, Cairo, Egypt \\ ${ }^{2}$ Department of Family Medicine, Ministry of Health, Cairo, Egypt \\ ${ }^{3}$ Department of Internal Medicine, Faculty of Medicine, Cairo University, Cairo, Egypt
}

Background: Contraceptive agents are widely used by women of reproductive age, and resulting depression is the most common side effect of this usage. This study aimed to study the effect of monthly injectable combined contraceptives versus that of combined oral contraceptive pills (COC) on patients' mood.

Methods: A prospective cohort study was conducted on 124 females aged 18-45 years attending the Kom-Ashfeen Family Medicine Unit, El-Kalyubia, Egypt. Participants were divided into three groups according to their choice: group A included 44 participants who received monthly combined injectable contraceptives (CIC); group B included 40 participants who took COC; and group C included 40 participants who used the copper intrauterine device (IUD). The Patient Health Questionnaire-9 (PHQ-9) score was assessed at the beginning of the study and after 6 months of follow-up.

Results: After 6 months of follow-up, there were mild but statistically significant increases in the PHQ-9 score in groups A and B, with group A (CIC users) showing the highest increase. Approximately $34.1 \%, 27.5 \%$, and $15 \%$ of CIC, COC, and IUD users, respectively, moved from the non-depression stage to mild depression after 6 months; this change was statistically significant in groups A and B only.

Conclusion: Monthly injectable combined contraceptives and oral contraceptive pills were associated with an increased risk of developing mild depression; this risk was higher in users of CICs, although the difference was not statistically significant. Thus, it is crucial to counsel patients about this possible risk and to follow them up. However, further studies are required to confirm our results.

Keywords: Depression; Ethinyl Estradiol; Levonorgestrel; Norethindrone; Hormonal Contraception

Received: May 11, 2020, Revised: July 16, 2020, Accepted: July 18, 2020

*Corresponding Author: Ghada M. Khafagy https://orcid.org/0000-0001-6941-7291

Tel: +20-01006393140, Fax: +20-023499141,E-mail: ghada.khafagy@kasralainy.edu.eg, ghadakhafajy@yahoo.com 


\section{INTRODUCTION}

Family planning can be used to delay pregnancy, especially in young mothers, thereby decreasing health-related problems and deaths in early childbearing. In addition, it can prevent high risk unintended pregnancies in older women. ${ }^{1)}$ For the last 50 years, daily combined oral contraceptive pills (COC) have been the favored short-acting hormonal contraceptives among most women. However, this system is an unsuitable or suboptimal choice for others. ${ }^{2)}$ Injectable contraception has been a popular contraceptive tool for several years. Currently, over 40 million women worldwide use injectable contraceptives to prevent pregnancy. ${ }^{3)}$ Combined injectable contraceptives (CIC) containing varying proportions of both estrogen and progestin were developed as a method of choice for women desiring the benefits of injectable contraception, while minimizing the side effects of progestin-only formulations. CIC have proven to be a highly effective and reversible method of birth control. ${ }^{4)}$

Although the use of combined hormonal contraception for women with mood disorders is not restricted and is considered to be safe, ${ }^{5)}$ the main cause of discontinuation of the combined contraceptive methods is the mood-related side effects. The percentage of women who complained of depressive mood, irritability, and anxiety ranges from $4 \%-10 \%$, and this problem can lead to lack of compliance, resulting in unintended pregnancies. ${ }^{6)}$ Therefore, mood monitoring and reporting of negative symptoms are advised, and women should consider alternative contraceptive methods accordingly, on an individual basis. ${ }^{5)}$

In 2020, the World Health Organization reported that women are more prone to depression than men, especially during the adolescence stage, which in turn leads to disabilities and increases the risk of suicide ${ }^{7}{ }^{7}$ therefore, it is important to know if any association exists between depressive symptoms and widely used contraceptive methods. ${ }^{8)}$ On reviewing the literature, we noticed that there was a lack of data on this area; accordingly, we aimed to estimate the severity of adverse mood in combined contraceptive use.

\section{METHODS}

This prospective cohort study was conducted from April 2018 to February 2019 on 128 women aged between 18-45 years old who presented to outpatient clinics seeking medical advice for contraception in the Kom-Ashfeen Family Medicine Unit, El-Kalyubia district, Egypt.

Participants were enrolled between April and August 2018. Women known to have thromboembolic diseases, hypertension, breast cancer (current or prior), diabetes, migraine with aura, and/or known psychiatric illnesses were excluded. Breastfeeding mothers and mothers who had delivered within the prior 6 months were excluded.

After initial enrollment of 208 patients according to the selection criteria, the physicians counseled the patients regarding the different contraceptive options, providing the advantages, disadvantages, and reliability of each different method; as a result of counseling, the patients individually decided which method to use. A total of 148 patients who chose to start using combined monthly injectable, combined oral pills, or an intrauterine device (IUD) were invited to participate in the study; 20 refused and 128 accepted. Those who accepted were divided into three groups according to their choice, as follows:

Group A included 48 participants who received monthly CIC; progesterone estrogen for hormonal contraception. Each $1 \mathrm{~mL}$ ampule contained $50 \mathrm{mg}$ norethisterone enanthate and $5 \mathrm{mg}$ estradiol valerate in oily solution. Forty-four patients completed the study, while four patients were lost to follow-up.

Group B included 40 participants who received COCs; progestin-estrogen combination for oral contraception; the contraceptive pack contains 21 tablets each with $0.15 \mathrm{mg}$ levonorgestrel and $0.03 \mathrm{mg}$ Ethinyl estradiol. All patients in this group completed the study.

The control group, $\mathrm{C}$, included 40 participants who used a copper $\mathrm{T}$ IUD, all of whom completed the study.

In the first visit, the medical history of all patients in the three groups was taken using the sheet of El-Gilany et al., ${ }^{9}$ which surveyed sociodemographic data, medical, menstrual, and obstetric history. This was followed by a clinical examination. Patients were assessed for depressive symptoms in their intermenstrual phase using the validated Arabic form of the Patient Health Questionnaire-9 (PHQ-9) before the start of the contraceptive method and 6 months later. ${ }^{10)}$ The PHQ-9 is a validated tool that assists clinicians in diagnosing depression and monitoring treatment responses. The nine items of the PHQ-9 are based directly on the nine diagnostic criteria for major depressive disorder in the Diagnostic and Statistical Manual of Mental Disorders, 4th edition; its internal consistency is 0.89 . A PHQ-9 score $\geq 10$ has a sensitivity of $88 \%$ and a specificity of $88 \%$ for major depression. PHQ-9 scores of 5, 10, 15, and 20 represented mild, moderate, moderately severe, and severe depression, respectively. ${ }^{11,12)}$

\section{Statistical Data Analysis}

All data were collected, tabulated, and statistically analyzed using IBM SPSS for Windows ver. 20.0 (IBM Corp., Armonk, NY, USA). Quantitative data are expressed as the mean \pm standard deviation and median (range), and qualitative data are expressed as absolute frequencies (number) and relative frequencies (percentages). A paired t-test was used to compare the pre- and post-results of normally distributed variables, while the Mann-Whitney U-test was used for non-normally distributed variables. One-way analysis of variance (ANOVA) was performed to compare more than two groups of normally distributed variables, while the Friedman ANOVA test was used for non-normally distributed variables. Categorical variables were compared using the chi-square test. All tests were two-sided. A P-value $<0.05$ was considered statistically significant, while a P-value $\geq 0.05$, was considered statistically insignificant.

\section{Ethical Considerations}

Ethical approval was obtained from the research committee of the Faculty of Medicine, Cairo University. Informed written consent was obtained from all patients after a full explanation of the nature of the 
study, benefits, and possible harms of the study.

\section{RESULTS}

Table 1 shows that there was no statistically significant difference between the three groups in terms of age and social class, ensuring homogeneity and matching of the three groups. Further, there was no statistically significant difference between the three studied groups in terms of the obstetric history (number of pregnancies $(\mathrm{P}=0.77)$, abortions $(\mathrm{P}=0.09)$, children $(\mathrm{P}=0.62)$, previous cesarean sections $(\mathrm{P}=0.49)$, normal labors $(\mathrm{P}=0.35)$, age of the oldest $(\mathrm{P}=0.93)$ and youngest son $(\mathrm{P}=0.98)$; and all participants' deliveries were performed under medical supervision and passed safely without any post-partum complications.

The majority of the women in this study had regular menses, moderate in quantity, and there was no statistically significant difference between the three studied groups in terms of menstrual history
$(\mathrm{P}=0.3)$, or the types of previously used contraceptive methods $(\mathrm{P}=0.51)$.

Table 2 shows that there were statistically significant differences between the three studied groups in terms of the PHQ-9 score after 6 months of follow-up $(\mathrm{P}<0.05)$, and the mean change in PHQ-9 score was higher in the combined injectable users, although this difference was not statistically significant compared to oral contraceptives $(\mathrm{P}=0.51)$. Table 3 shows that there were statistically significant differences between the pre- and post-results of groups A and B regarding PHQ-9 score after 6 months of follow-up $(\mathrm{P}<0.05)$. As shown in the boxplot graph in Figure 1, there were statistically significant differences between groups A and B in terms of the PHQ-9 score after 6 months of follow-up $(\mathrm{P}<0.05)$.

Figure 2 shows that before the study, there were no statistically significant differences among the studied groups regarding PHQ-9 score categories. The largest percentage of participants had no depression; $77.3 \%$ of the women in group A (COC users), $75 \%$ of the women in

Table 1. Socio-demographic characteristics of the study subjects

\begin{tabular}{|c|c|c|c|c|}
\hline Characteristic & Group A (CIC users) $(n=44)$ & Group B (COC users) $(n=40)$ & Group C (IUD users) $(n=40)$ & P-value \\
\hline Age $(y)$ & & & & $0.46^{\star}$ \\
\hline $18-24$ & $10(22.7)$ & $9(22.5)$ & $8(20.0)$ & \\
\hline $25-34$ & $18(40.9)$ & $20(50.0)$ & $24(60.0)$ & \\
\hline $35-45$ & $16(36.4)$ & $11(27.5)$ & $8(20.0)$ & \\
\hline Socioeconomic status categories & & & & $0.12^{*}$ \\
\hline High (64-84) & $38(86.4)$ & $34(85.0)$ & $28(70.0)$ & \\
\hline Middle (43-63) & $6(13.6)$ & $6(15.0)$ & $12(30.0)$ & \\
\hline $\operatorname{Low}(\leq 42)$ & 0 & 0 & 0 & \\
\hline Regularity of menstruation & & & & $0.3^{*}$ \\
\hline Regular & $40(36.0)$ & $33(29.7)$ & $38(34.2)$ & \\
\hline Irregular & $4(33.3)$ & $6(50.0)$ & $2(16.7)$ & \\
\hline Length of menstrual flow (d) & & & & $0.63^{*}$ \\
\hline$\leq 4$ & $37(36.3)$ & $34(33.3)$ & $31(30.4)$ & \\
\hline$>4$ & $7(31.8)$ & $6(27.3)$ & $9(40.9)$ & \\
\hline No. of pregnancies & $2.91 \pm 1.27$ & $3.1 \pm 1.37$ & $2.98 \pm 0.97$ & $0.77^{\dagger}$ \\
\hline No. of abortions & $0.25 \pm 0.53$ & $0.43 \pm 0.96$ & $0.1 \pm 0.3$ & $0.09^{\dagger}$ \\
\hline No. of children & $2.68 \pm 1.01$ & $2.78 \pm 1.16$ & $2.9 \pm 0.87$ & $0.62^{\dagger}$ \\
\hline Age of the oldest child (y) & $9.72 \pm 6.38$ & $9.29 \pm 5.14$ & $9.29 \pm 6.38$ & $0.93^{\dagger}$ \\
\hline Age of the youngest child (y) & $4.07 \pm 3.58$ & $3.99 \pm 2.77$ & $3.89 \pm 4.88$ & $0.98^{\dagger}$ \\
\hline No. of deliveries & $2.48 \pm 1.04$ & $2.58 \pm 1.15$ & $2.8 \pm 0.91$ & $0.35^{\dagger}$ \\
\hline No. of cesarean sections & $0.91 \pm 1.03$ & $1.08 \pm 1.21$ & $1.23 \pm 1.4$ & 0.49 \\
\hline No. of normal labors & $1.5 \pm 1.45$ & 1.62 & 1.43 & 0.97 \\
\hline
\end{tabular}

Values are presented as mean \pm standard deviation or number (\%). Socioeconomic status were assessed according to the scale of El-Gilanny et al..$^{9)}$ and classified into low $\leq 42$, middle 43-63, and high 64-84.

CIC, combined injectable contraceptives; COC, combined oral contraceptives; IUD, intrauterine device.

*By chi-square test. 'By one-way analysis of variance test.

Table 2. Comparison between the PHQ-9 score in the studied groups before the study and after 6 months of follow-up

\begin{tabular}{|c|c|c|c|c|c|c|c|c|c|c|c|}
\hline & \multicolumn{4}{|c|}{ Before } & \multicolumn{4}{|c|}{ After } & \multicolumn{3}{|c|}{ P-value } \\
\hline & Group A (CIC) & Group B (COC) & Group C (IUD) & $P$-value & Group $\mathrm{A}(\mathrm{CIC})$ & Group B (COC) & Group C (IUD) & P-value & Group A-C & Group B-C & Group A-B \\
\hline PHQ-9 & $3.6 \pm 1.56$ & $3.6 \pm 2.03$ & $3.2 \pm 1.8$ & $0.3^{*}$ & $5.2 \pm 2.1$ & $4.6 \pm 2.03$ & $3.7 \pm 1.6$ & $0.003^{*}$ & $0.001^{\dagger}$ & $0.008^{\dagger}$ & $0.195^{\dagger}$ \\
\hline
\end{tabular}

Values are presented as mean \pm standard deviation.

PHQ-9, Patient Health Questionnaire-9; CIC, combined injectable contraceptives; COC, combined oral contraceptives; IUD, intrauterine device.

*By one-way analysis of variance test. ${ }^{\dagger}$ By unpaired t-test. 
Table 3. Comparison between pre and post results of PHQ-9 within each of the three groups

\begin{tabular}{|c|c|c|c|c|c|c|c|c|c|}
\hline & \multicolumn{3}{|c|}{ Group A (CIC users) } & \multicolumn{3}{|c|}{ Group B (COC users) } & \multicolumn{3}{|c|}{ Group C (IUD users) } \\
\hline & Pre & Post & $P$-value & Pre & Post & $\mathrm{P}$-value & Pre & Post & $P$-value \\
\hline PHQ-9 & $3.6 \pm 1.65$ & $5.2 \pm 2.1$ & $<0.001^{\star}$ & $3.6 \pm 2.03$ & $4.6 \pm 2.03$ & $0.001^{*}$ & $3.2 \pm 1.8$ & $3.7 \pm 1.6$ & $0.29^{*}$ \\
\hline
\end{tabular}

Values are presented as mean \pm standard deviation. A P-value $<0.05$ is statistically significant. PHQ-9 scores of 5, 10, 15, and 20 represented mild, moderate, moderately severe, and severe depression, respectively.

PHQ-9, Patient Health Questionnaire-9; CIC, combined injectable contraceptives; COC, combined oral contraceptives; IUD, intrauterine device.

*By paired t-test.

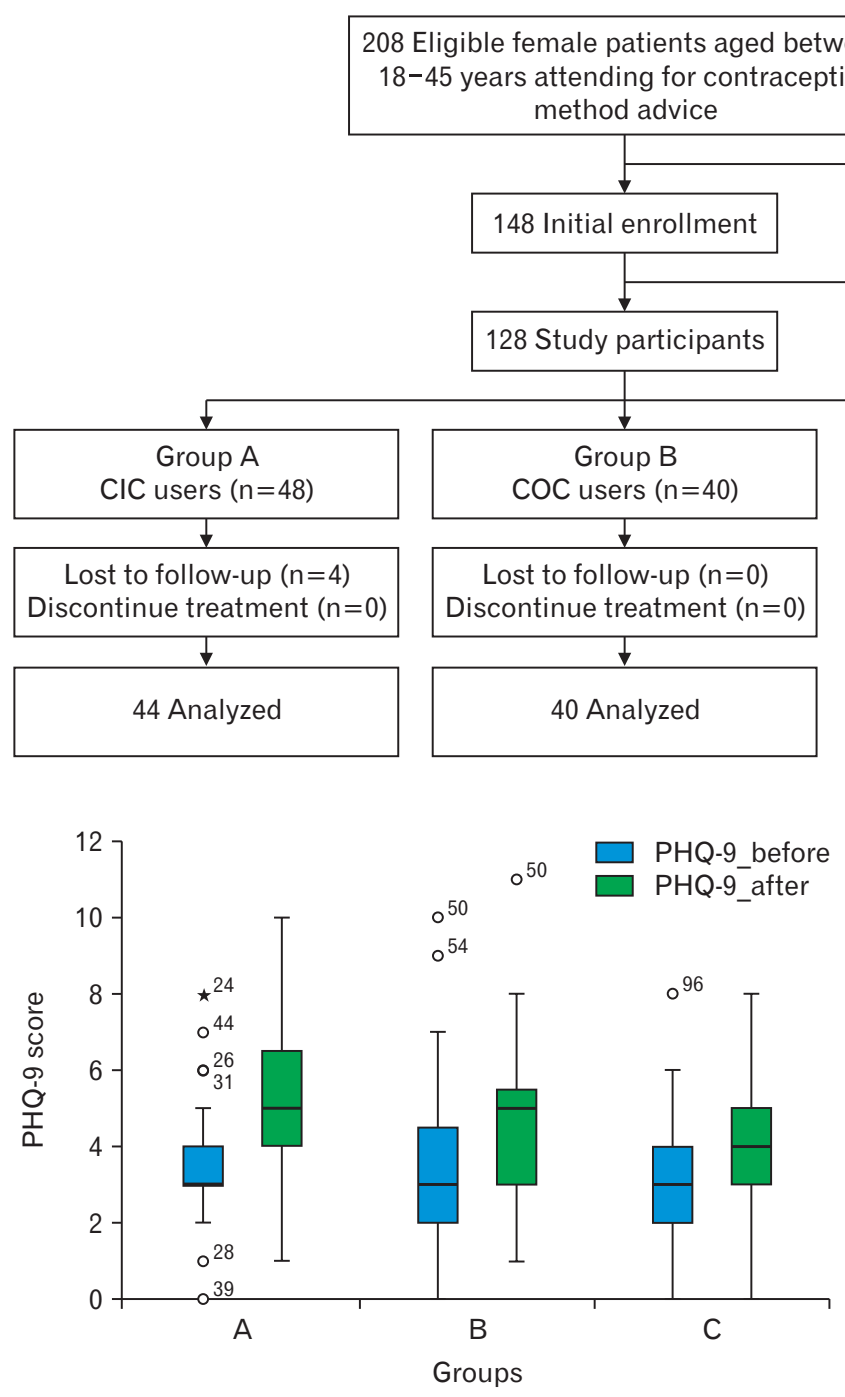

Figure 2. Comparison between the Patient Health Questionnaire-9 (PHQ-9) scores in the studied groups before the study and after 6 months of follow-up. The symbol ${ }^{\circ}$ indicates possible outliers and the symbol $\star$ indicates the most probable outliers.

group B (CIC users), and $87.5 \%$ of the women in group C (IUD users) did not have depression, while $22.7 \%, 22.5 \%$, and $12.5 \%$ of women in groups A, B, and C had mild depression, respectively.

Figure 3 shows that after 6 months of follow-up, $54.5 \%$ of the women in group A, $50 \%$ of the women in group B, and $27 \%$ of the women in group $\mathrm{C}$ became mildly depressed; these increases were statistically significant in group $\mathrm{A}(\mathrm{P}=0.006)$ and group $\mathrm{B}(\mathrm{P}=0.03)$.
60 Chose other methods

20 Refused

to participate

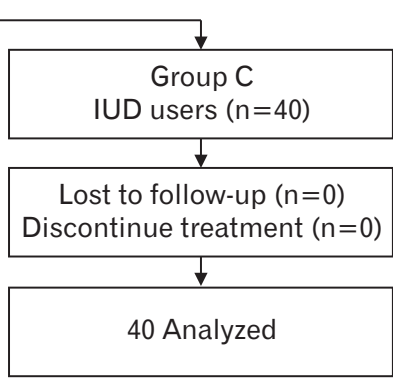

Figure 1. Flow chart demonstrating the selection of study participants. CIC, combined injectable contraceptives; COC, combined oral contraceptives; IUD, intrauterine device.

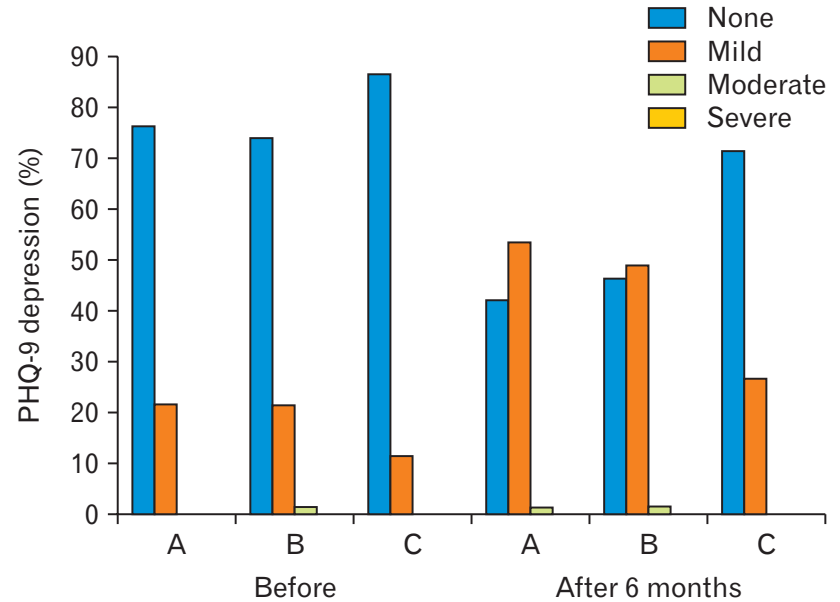

Figure 3. Comparison among the studied groups regarding Patient Health Questionnaire-9 (PHQ-9) depression categories before and after 6 months. P-value for group A (combined injectable contraceptives users) is 0.006 ; for group B (combined oral contraceptives users) is 0.03 ; and for group $\mathrm{C}$ (intrauterine device users) is 0.09 .

\section{DISCUSSION}

The findings of this study showed that there were statistically significant mild increases in the PHQ-9 scores of CIC users and COC users after the 6-month follow-up period (Table 3). Although there was no 
statistically significant difference between the groups in PHQ-9 score at the beginning of the study, after the 6-month follow-up period, a statistically significant difference $(\mathrm{P}<0.05)$ was observed, where group A, monthly combined injectable contraceptive users, had the highest mean value $(5.2 \pm 2.1)$, followed by group B, the COC users, $(4.6 \pm 2.03)$ (Table 2).

Previous studies have revealed that emotion regulation areas such as the amygdala and the hypothalamus contain estrogenic receptors, and animal studies have shown that both estrogen and progesterone modulate the synthesis of neurotransmitters, such as serotonin. ${ }^{13)}$ The types of synthetic progestins vary and can cross the blood-brain barrier. ${ }^{14)}$ Other researchers have found that oral contraceptive users have blunted responses to stress tests. ${ }^{15)}$

Consistent with our study, Anderl et al. ${ }^{16)}$ reported that there is a long-term association between adolescent oral contraceptive use and depression risk in adulthood, regardless of current oral contraceptive use, suggesting that adolescence may be a sensitive period during which oral contraceptive use could increase women's risk of depression, even years after the first exposure. In addition, de Wit et al. ${ }^{17)}$ in 2020 concluded, in a prospective cohort study conducted to investigate the association between oral contraceptive use and depressive symptoms, that although oral contraceptive use showed no association with depressive symptoms, the monitoring of depressive symptoms in adolescents who use oral contraceptives is important, as the use of oral contraceptives may affect their quality of life and put them at risk of non-adherence.

According to Lundin et al. ${ }^{6}$ in 2016, COC use is associated with small but statistically significant mood side effects during the intermenstrual phase. These findings are driven by a subgroup of women who clearly suffer from COC-related side effects. However, positive mood effects were noted in the premenstrual phase, and the proportion of women with clinically relevant mood worsening did not differ between the different treatment groups. Conversely, a double-blinded randomized placebo-controlled trial conducted by Zethraeus et al. ${ }^{18)}$ in 2017 found that COC had no effect on depressive mood.

After the 6-month follow-up period in our study, approximately $34.1 \%$ and $27.5 \%$ of the study groups moved from the non-depression stage to mild depression in CIC and COC users, respectively.

Hall et al. ${ }^{19)}$ in 2015 in his review article suggested that modern contraceptives with a lower dosage of steroids do not have a clinically relevant impact on women's mood, as compared to higher doses of steroids that were used during the 1970s when depression was a known side effect of oral contraceptives use.

In agreement with our study, a prospective cohort study conducted by Skovlund et al. ${ }^{20)}$ in 2016 on women and adolescents aged 15 to 34 years in Denmark with no history of prior depression diagnosis, found that those who used hormonal contraceptives had a greater risk of developing depression within 6 months of using contraceptives than those who did not use contraceptives. They thus concluded that the use of hormonal contraception, especially rings, patches, or progesterone-only forms, was associated with the subsequent use of antidepres- sants, and the first diagnosis of depression, suggesting depression as a potential adverse effect of hormonal contraceptive use, particularly among adolescents.

Although some of these contraceptive methods are combined, differences in side effects according to the methods of delivery have been; for example, the risk of depression is higher with long-acting reversible contraceptive methods than with oral contraceptive pills. Consequently, different kinds of hormonal contraceptives containing different doses, types, and ratios of estrogen and progestin may affect the mode and central nervous system differently. Our study suggests that only some women are susceptible to these side effects, but we could not determine which group is more prone to depression; thus, medical care providers need to be aware of this possible risk, and provide counseling in addition to following up our patients effectively.

Although not significantly different, in our study the risk of depression was higher in combined injectable users that in those who used oral contraceptives. This knowledge would help medical care workers to choose the most suitable contraceptive methods for susceptible patients, while providing patients with more comprehensive information regarding the safety concerns of using these methods, especially for patients who were previously diagnosed or had received treatment for major depression.

\section{Limitations and Strength}

This study had several limitations. First, the sample size was small, although it was calculated to be sufficient for statistical analysis. Second the study was conducted only on patients at the Family Centre Outpatient Clinic. Although these outpatients have a high flow rate, they are considered to be one focal zone. Further, this study was not blinded because it depended on the patients' right to choose the most suitable contraceptive methods.

To our knowledge, this study is the first to investigate the effects of combined injectable contraceptive methods on depression. Participants in the three groups were well matched in terms of age, socioeconomic level, menstrual history, and obstetric history.

\section{Implications for Practice and/or Research}

Given that ICI users showed a higher increase in PHQ-9 score than IUD users, more studies are needed to evaluate the effects on patients who have been receiving depression treatment or who have a previous history of depression.

\section{CONFLICT OF INTEREST}

No potential conflict of interest relevant to this article was reported.

\section{ORCID}

Ghada M. Khafagy: https://orcid.org/0000-0001-6941-7291

Hebatallah L. Shalaby: https://orcid.org/0000-0003-0868-2897

Nagwa E. Saad: https://orcid.org/0000-0001-6309-6757 
Marwa D. Hasan: https://orcid.org/0000-0003-0422-109X

\section{REFERENCES}

1. World Health Organization. Selected practice recommendations for contraceptive use. 3rd ed. Geneva: World Health Organization; 2016.

2. Mansour D. International survey to assess women's attitudes regarding choice of daily versus nondaily female hormonal contraception. Int J Womens Health 2014;6:367-75.

3. Halpern V, Stalter RM, Owen DH, Dorflinger LJ, Lendvay A, Rademacher KH. Towards the development of a longer-acting injectable contraceptive: past research and current trends. Contraception 2015;92:39.

4. Dimkpa OJ, Okwudili OE. Experience with combined injectable contraceptive (Norigynon) in Port Harcourt. J Adv Med Med Res 2017;19: 1-6.

5. World Health Organization. Medical eligibility criteria for contraceptive use. 5th ed. Geneva: World Health Organization; 2015.

6. Lundin C, Danielsson KG, Bixo M, Moby L, Bengtsdotter H, Jawad I, et al. Combined oral contraceptive use is associated with both improvement and worsening of mood in the different phases of the treatment cycle: a double-blind, placebo-controlled randomized trial. Psychoneuroendocrinology 2017;76:135-43.

7. World Health Organization. Depression fact sheet [Internet]. Geneva: World Health Organization; 2020 [cited 2020 Feb 5]. Available from: https://www.who.int/news-room/fact-sheets/detail/depression.

8. Smith K, Nayyar S, Rana T, Archibong AE, Looney KR, Nayyar T. Do progestin-only contraceptives contribute to the risk of developing depression as implied by beta-arrestin 1 levels in leukocytes?: a pilot study. Int J Environ Res Public Health 2018;15:1966.

9. El-Gilany A, El-Wehady A, El-Wasify M. Updating and validation of the socioeconomic status scale for health research in Egypt. East Mediterr Health J 2012;18:962-8.

10. AlHadi AN, AlAteeq DA, Al-Sharif E, Bawazeer HM, Alanazi H,
AlShomrani AT, et al. An Arabic translation, reliability, and validation of Patient Health Questionnaire in a Saudi sample. Ann Gen Psychiatry 2017;16:32.

11. Kroenke K, Spitzer RL. The PHQ-9: a new depression diagnostic and severity measure. Psychiatr Ann 2002;32:509-15.

12. Kroenke K, Spitzer RL, Williams JB. The PHQ-9: validity of a brief depression severity measure. J Gen Intern Med 2001;16:606-13.

13. Toffoletto S, Lanzenberger R, Gingnell M, Sundstrom-Poromaa I, Comasco E. Emotional and cognitive functional imaging of estrogen and progesterone effects in the female human brain: a systematic review. Psychoneuroendocrinology 2014;50:28-52.

14. Pluchino N, Cubeddu A, Giannini A, Merlini S, Cela V, Angioni S, et al. Progestogens and brain: an update. Maturitas 2009;62:349-55.

15. Rohleder N, Wolf JM, Piel M, Kirschbaum C. Impact of oral contraceptive use on glucocorticoid sensitivity of pro-inflammatory cytokine production after psychosocial stress. Psychoneuroendocrinology 2003;28:261-73.

16. Anderl C, Li G, Chen FS. Oral contraceptive use in adolescence predicts lasting vulnerability to depression in adulthood. J Child Psychol Psychiatry 2020;61:148-56.

17. De Wit AE, Booij SH, Giltay EJ, Joffe H, Schoevers RA, Oldehinkel AJ. Association of use of oral contraceptives with depressive symptoms among adolescents and young women. JAMA Psychiatry 2020;77:52-9.

18. Zethraeus N, Dreber A, Ranehill E, Blomberg L, Labrie F, von Schoultz $\mathrm{B}$, et al. A first-choice combined oral contraceptive influences general well-being in healthy women: a double-blind, randomized, placebocontrolled trial. Fertil Steril 2017;107:1238-45.

19. Hall KS, Steinberg JR, Cwiak CA, Allen RH, Marcus SM. Contraception and mental health: a commentary on the evidence and principles for practice. Am J Obstet Gynecol 2015;212:740-6.

20. Skovlund CW, Mørch LS, Kessing LV, Lidegaard O. Association of hormonal contraception with depression. JAMA Psychiatry 2016;73:115462. 